\title{
Diagnóstico pela Ecocardiografia Transesofágica e Evolução de 35 Pacientes Portadores de Flail Mitral Valve
}

\author{
Mônica M. C. Caldas, Lúcia M. A. Fenelon, William A. M. Esteves, Tamara Katina, \\ Nilson Maia, Márcia M. Barbosa
}

Belo Horizonte, MG

\begin{abstract}
Objetivo-Descrever os achados ao ecocardiograma transesofágico e evolução clínica de portadores de flail mitral valve.

Métodos - No periodo de janeiro/93 a março/97, 1675 pacientes foram submetidos, em nossa instituição, a ecocardiograma transesofágico, sendo que em 35 casos foi feito o diagnóstico de flail mitral valve e, posteriormente, obtida sua evolução clínica.

Resultados - A idade dos pacientes variou 12 a 87

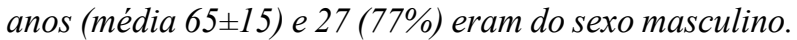
O folheto posterior foi o mais acometido (25 pacientes, $71 \%$ ). O mecanismo do flail foi ruptura de cordoalha tendinea em todos os casos, exceto um, que apresentava importante alongamento e redundância de cordoalha. $A$ etiologia foi prolapso elou degeneração mixomatosa em 15 pacientes, degenerativa em 9, isquêmica em 5 , reumática em 4 e endocardite em 3. Regurgitação mitral de grau importante ocorreu em 25 (71\%) pacientes e moderada em 10 (29\%). O tempo médio de acompanhamento foi de $375 \pm 395$ dias (1 a 1380). Foram submetidos a tratamento clínico 19 pacientes e a tratamento cirúrgico 16, sendo que em todos foi confirmado o diagnóstico transesofágico. $O$ número total de óbitos (hospitalar e pós-hospitalar) foi alto $(34 \%)$, tanto em pacientes submetidos a tratamento clínico quanto cirúrgico. Entre os sobreviventes, 17 estão em classe funcional (CF) I e 6 em CF II da NYHA.

Conclusão - O diagnóstico de flail mitral valve ao ecocardiograma transesofágico é acurado, permitindo a definição de sua etiologia emecanismo. A alta mortalidade à época do diagnóstico, provavelmente, se relaciona à gravidade da doença subjacente. Embora os pacientes não operados estejam evoluindo bem, a baixa CF observada nestes pacientes pode ser atribuída ao curto periodo de seguimento.
\end{abstract}

Palavras-chave: ruptura de cordoalha, regurgitação mitral, ecocardiograma transesofágico

\section{Flail Mitral Valve Leaflet Diagnosed by Transesophageal Echocardiography. Report and Evolution of 35 Cases}

Purpose - To describe transesophageal echocardiographic findings in patients presenting with flail mitral valve and to obtain their clinical follow-up.

Methods - From January/93 to March/97, 1675 patients were submitted to transesophageal echocardiogram at our institution; 35 of them were diagnosed as having flail mitral valve and their clinical follow-up was obtained.

Results - Thirty-five patients with the diagnosis offlail mitral valve were studied. Mean age was $65 \pm 15$ years (1287) and $27(77 \%)$ were male. The posterior leaflet was involved in $25(71 \%)$ patients. Ruptured chordae tendineae was present in all but one patient in whom flail mitral valve was due to a very elongated and redundant chordae. The etiology was prolapse and/or mixomatous degeneration in 16 patients, degenerative in 10, ischemic in 5, rheumatic in 4 and endocarditis in 3. Mitral regurgitation was severe in $25(71 \%)$ patients and moderate in $10(29 \%)$. The mean follow-up was $375 \pm 395$ days (1 to 1380). Nineteen patients were followed clinically and 16 were submitted to surgery (transesophageal echocardiogram findings were confirmed in all 16). Overall mortality was high (34\%). Among the survivors, 17 are in NYHA class I and 6 in class II.

Conclusion - The diagnosis of flail mitral valve by transesophageal echocardiography is accurate, allowing for the definition of its mechanism and etiology, as well as the evaluation of hemodynamic compromise. The observed high mortality at the time of diagnosis may be related to the severity of the disease causing the flail mitral valve. Although non-operated survivors are doing well, the observed low functional class in these patients may be related to the short period offollow-up.

Key-words: flail mitral valve, mitral regurgitation, transesophageal echocardiography

Arq Bras Cardiol, volume 71 (nº 6), 763-767, 1998

Ecocenter - Serviço de Ecocardiografia dos Hospitais Socor e Vera Cruz - Belo Horizonte

Correspondência: Márcia M. Barbosa - Rua Jornalista Djalma Andrade, 210 30320-540 - Belo Horizonte, MG

Recebido para publicação em 19/3/98

Aceito em 20/8/98
Ruptura de cordoalha tendínea da valva mitral foi descrita em $1806^{1}$, porém, apenas recentemente sua freqüência como causa de doença valvar mitral, tem sido apreciada. A ruptura de cordoalha com conseqüente falha na coaptação dos folhetos representa hoje a causa mais comum de 
regurgitação mitral pura em países desenvolvidos ${ }^{2}$. A etiologia mais freqüente é a degeneração mixomatosa ${ }^{1}$, seguindo-se a endocardite e a doença coronária. $\mathrm{O}$ folheto posterior é freqüentemente o mais acometido, com estudos demonstrando seu acometimento em até $83 \%$ dos casos ${ }^{1,3,4}$.

$\mathrm{O}$ ecocardiograma constitui-se em uma importante modalidade diagnóstica desta doença. O diagnóstico de flail mitral valve por ruptura de cordoalha já era feito desde a época do modo $\mathrm{M}$, que mostrava ecos sistólicos no átrio esquerdo (AE), vibrações sistólicas ou diastólicas dos folhetos da valva mitral e movimentação exagerada e/ou paradoxal do folheto posterior da valva mitral ${ }^{2}$. Posteriormente, o ecocardiograma bidimensional acrescentou muito ao diagnóstico, permitindo a visualização da movimentação anômala do folheto dentro do AE e conseqüente falha de coaptação da valva mitral ${ }^{2}$. Contudo, devido à proximidade do esôfago ao AE, o ecocardiograma transesofágico (ETE) permite uma melhor exploração da cavidade atrial e, portanto, esta metodologia mostra-se mais sensível e específica no diagnóstico da ruptura da valva mitral do que o ecocardiograma transtorácico $(\mathrm{ETT})^{2,3}$, permitindo o diagnóstico acurado doflail e de seu mecanismo subjacente. Na imensa maioria dos casos, este se dá por ruptura de cordoalha e, apenas excepcionalmente, por cordoalhas alongadas e redundantes, porém sem ruptura ${ }^{3}$.

As cordas tendíneas servem para sustentação da valva mitral. A ruptura destas cordas pode ocorrer, espontaneamente, ou ser secundária a uma variedade de doenças cardíacas, como a endocardite infecciosa. O resultado é um folheto com movimentação caótica, que ocorre geralmente de forma aguda, com conseqüente regurgitação mitral hemodinamicamente significativa ${ }^{6}$. A gravidade da regurgitação correlaciona-se à localização e número de cordas tendíneas envolvidas, assim como à presença de doença prévia da valva mitral.

Ao contrário do que ocorre em pacientes com regurgitação mitral reumática crônica, aqueles com regurgitação secundária à ruptura de cordoalha apresentam alta mortalidade no primeiro ano $^{6}$. Esses pacientes tipicamente se apresentam em insuficiência cardíaca congestiva (ICC) rapidamente progressiva e elevação da pressão capilar pulmonar com onda " $v$ " elevada, requerendo intervenção cirúrgica precoce. Porém, ocasionalmente a ruptura pode não se associar a uma deterioração hemodinamicamente significativa, pelo menos inicialmente, o que pode ser atribuído ao grau de regurgitação mitral que, por sua vez, é influenciado por fatores como posição, localização e número de cordas rotas, processo patológico subjacente, complacência do $\mathrm{AE}$ e função sistólica do ventrículo esquerdo (VE) ${ }^{7}$. A ruptura de cordas terciárias também foi proposta como um provável mecanismo nos casos sem descompensação hemodinâmica ${ }^{7}$.

A regurgitação mitral relacionada à ruptura de folhetos geralmente é excêntrica. O ETE é capaz de detectar a regurgitação mitral em todos os pacientes, com sensibilidade de $100 \%$ e, embora a detecção da regurgitação mitral seja acurada também ao ETT, pode haver discrepâncias entre estas duas metodologias, principalmente na quantificação da regurgitação mitral leve ou moderada ${ }^{8}$. Além disto, a capacidade do ETE em se destacar fluxo reverso em veia pulmonar nos casos de regurgitação mitral significativa torna esta metodologia altamente atrativa na avaliação e quantificação da regurgitação mitral ${ }^{9}$.

Assim, o presente estudo tem como objetivo descrever os achados à ETE em pacientes diagnosticados em nossa instituição como portadores de flail mitral valve.

\section{Métodos}

Trata-se de estudo parcialmente retrospectivo, realizado no período de janeiro/93 a março/97, quando 1675 ETEs foram realizados em nossa instituição, sendo que 35 apresentavam critérios diagnósticos de flail mitral valve.

Todos os pacientes foram submetidos à ETE sob sedação com midazolam e com monitorização da freqüência cardíaca. Nenhum paciente apresentou complicações relacionadas ao exame. Equipamento HP Sonos 1000 ou 2000, com sonda transesofágica biplanar, foi utilizado em todos os casos e obtidos os cortes convencionais ${ }^{10}$. O diagnóstico de flail mitral valve era feito ao se detectar a presença de uma coaptação anormal dos folhetos em sístole, com movimentação caótica de um dos folhetos dentro de AE, geralmente associada à ruptura de cordoalha e acompanhada de regurgitação mitral significativa. Este achado era sempre observado em todos os cortes do ETE em que a valva mitral podia ser visualizada. O aspecto da valva mitral era analisado, no sentido de se detectar alterações associadas sugestivas de doenças subjacentes (prolapso, acometimento reumático, endocardite, e outros). A quantificação da regurgitação mitral era feita tambémem ambos os cortes ortogonais doETE, a fim de se teruma avaliação espacial do jato e de sua excentricidade.

Os critérios para indicação de tratamento clínico ou cirúrgico dependeram exclusivamente do médico assistente. A cirurgia foi realizada em nosso serviço e a técnica utilizada foi a troca valvar. Os dados ecocardiográficos foram comparados aos achados cirúrgicos nos 16 pacientes submetidos à cirurgia.

Evolução clínica foi obtida em todos os pacientes através do acompanhamento durante a fase hospitalar (nos casos de morte intra-hospitalar) ou posteriormente, através de entrevista telefônica do paciente e/ou de seu assistente, realizada em um único tempo para todos os pacientes do estudo.

\section{Resultados}

Dos 35 pacientes com o diagnóstico de flail mitral valve, 27 (77\%) eram do sexo masculino e as idades variaram de 12 a 87 (média $65 \pm 15$ ) anos. O folheto posterior foi o mais freqüentemente envolvido, encontrando-se acometido em $25(71 \%)$ pacientes. Esta maior predominância do folheto posterior está de acordo com os dados da literatura que mostraram acometimento deste folheto em até $83 \%$ dos ca$\operatorname{sos}^{1,3,4}$. A ruptura de cordoalha (fig. $1 \mathrm{e} 2$ ) ocorreu em todos os pacientes, exceto um em que, embora não fosse detectada corda rota, havia nítida má coaptação dos folhetos e 


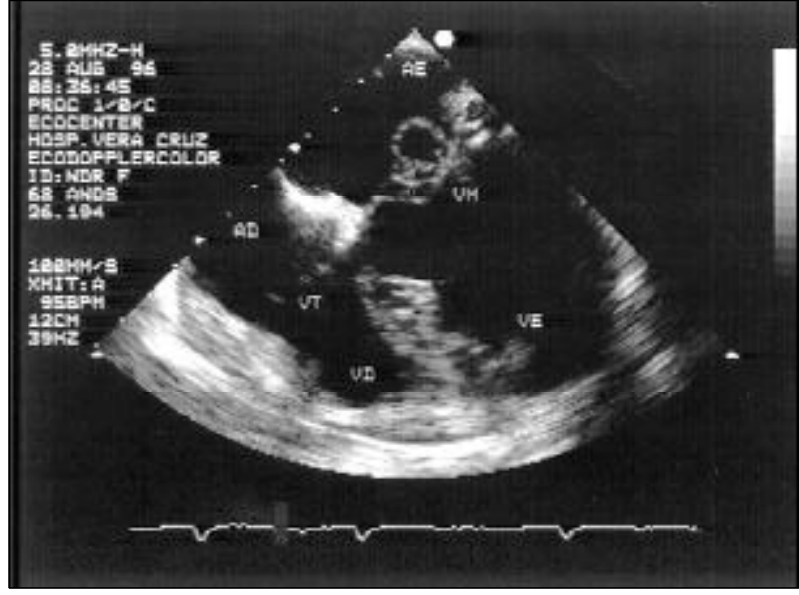

Fig. 1 - Corte de 4 câmaras ao ecocardiograma transesofágico mostrando importante prolapso do folheto posterior para o átrio esquerdo por ruptura de cordoalha. AD átrio direito; $\mathrm{AE}$ - átrio esquerdo; VD - ventrículo direito; VE - ventrículo esquerdo; VM - valva mitral; VT - valva tricúspide.

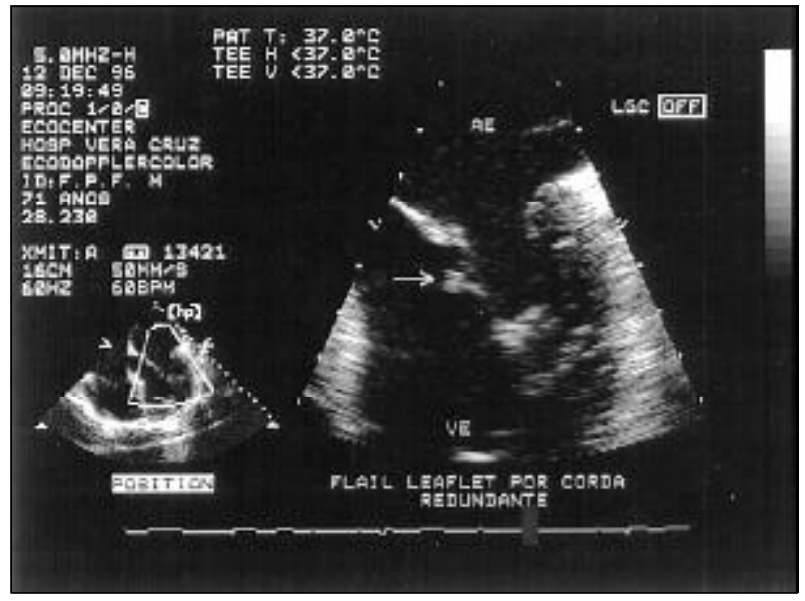

Fig. 3 - "Zoom" da valva mitral em corte de 4 câmaras do ecocardiograma transesofágico, mostrando a redundância da cordoalha e prolapso do folheto anterior para o átrio esquerdo. AE- átrio esquerdo; VE- ventrículo esquerdo.

prolapso do folheto anterior para dentro da cavidade atrial, devido a uma corda nitidamente redundante e alongada (fig. 3 e 4). Este folheto não apenas prolapsava para o $\mathrm{AE}$, mas apresentava movimentação caracteristicamente anômala de sua porção distal, diferenciando-se, portanto, do diagnóstico de prolapso convencional da valva mitral.

Quanto à etiologia, o aspecto da valva mitral permitiu que o diagnóstico etiológico fosse inferido: prolapso, associado ou não à degeneração mixomatosa, ocorreu em 16 pacientes, alterações degenerativas em 10, isquêmicas em cinco, reumáticas em quatro e presença de vegetações em três. Estes dados são semelhantes aos achados da literatura, que mostram prevalência do prolapso como causa etiológica mais freqüente ${ }^{1,2,11,12}$.

Regurgitação mitral excêntrica ocorreu em todos os pacientes, sendo importante em $25(71 \%)$ e moderada em 10. Dos 25 pacientes com regurgitação mitral importante, 12 foram submetidos a tratamento clínico e 13 a tratamento cirúrgico. Dos 10 pacientes com regurgitação mitral moderada,

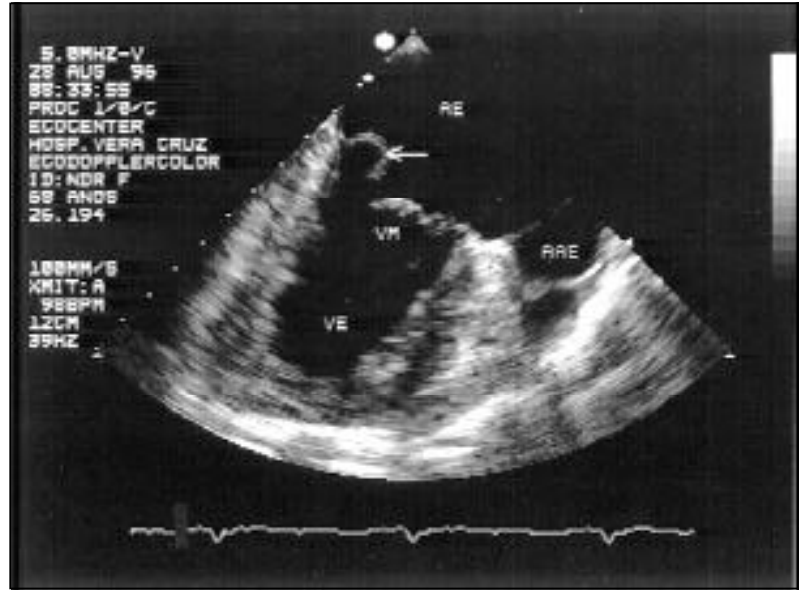

Fig. 2 - Mesmo caso da figura 1, mostrando o flail ao corte longitudinal do ecocardiograma transesofágico. $\mathrm{AE}$ - átrio esquerdo; VE - ventrículo esquerdo.

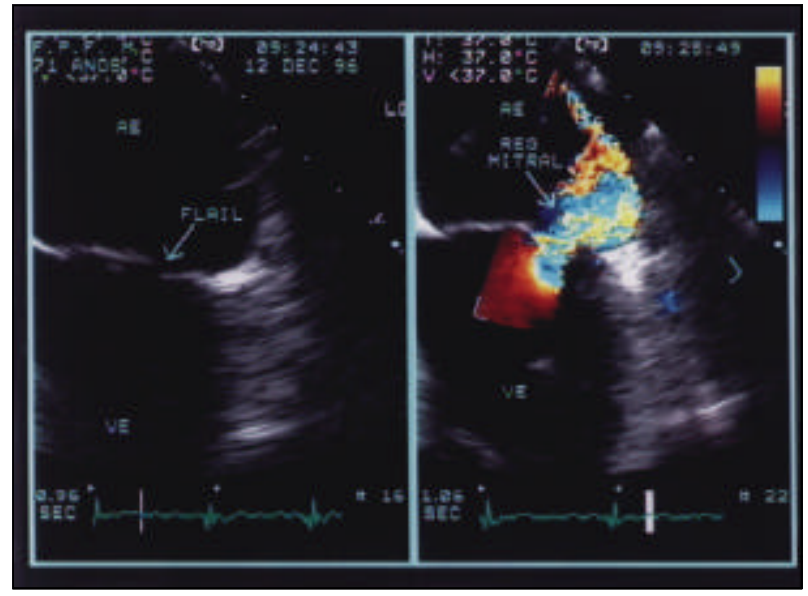

Fig. 4 - Corte de 4 câmaras ao ecocardiograma transesofágico do mesmo caso da figura 3, mostrando regurgitação mitral de grau moderado do Doppler de fluxo a cores. AEátrio esquerdo; VE- ventrículo esquerdo.

sete foram submetidos a tratamento clínico e três a tratamento cirúrgico. Vegetações e derrame pericárdico ocorreram em três pacientes, respectivamente.

O tempo médio de evolução foi de $375 \pm 395$ dias, variando de um dia (paciente com abscesso cerebral e grande vegetação em valva mitral que evoluiu rapidamente para o óbito) a 1380 dias. Dezenove pacientes foram submetidos a tratamento clínico, dos quais nove faleceram. A causa de óbito desses pacientes foi: abdome agudo em um, choque cardiogênico em um, pneumonia em um, insuficiência coronária em um, complicação pós-operatória em um (cirurgia de fratura de fêmur) e desconhecida em quatro. Seis desses nove tiveram morte intra-hospitalar (nos primeiros 30 dias após o diagnóstico), refletindo a gravidade do quadro inicial e, provavelmente, da doença subjacente (endocardite e suas complicações).

Dezesseis pacientes foram submetidos a tratamento cirúrgico e três desses evoluíram para óbito. As causas de óbito foram: desconhecida em dois pacientes e choque 
cardiogênico em um. Os achados cirúrgicos nos 16 pacientes foram todos compatíveis com o diagnóstico ecocardiográfico: prolapso em oito, alterações degenerativas em três, reumática em dois, isquêmica em dois e endocardite em um.

Dentre os sobreviventes submetidos a tratamento clínico (10), sete estão em classe funcional (CF) I e três em CF II da NYHA. As causas de ruptura de cordoalha nesses sete pacientes foram: alterações degenerativas em três, prolapso em dois, reumática em um e isquêmica em um. O tratamento medicamentoso desses pacientes foi o habitual na regurgitação mitral. Em relação aos pacientes submetidos à cirurgia que sobreviveram (13), 11 estão em CF I e, dois em CF II. Os dados dos 35 pacientes encontram-se na tabela I.

\section{Discussão}

O ETE permite uma análise acurada da anatomia da valva mitral, permitindo que sejam feitas, inclusive, inferências sobre sua etiologia. Complementa oETT no diagnóstico de flail mitral valve principalmente nos exames tecnicamente difíceis. Permite a visualização adequada da má coaptação dos folhetos da valva mitral e da ruptura de cordoalha, que representam o dado mais específico para o diagnóstico de flail mitral valve. Além disso, tanto o grau quanto a excentricidade do jato de regurgitação mitral podem ser adequadamente estudados. A regurgitação mitral, principalmente quando excêntrica, constitui-se em um dado sensível para o diagnóstico do flail ${ }^{2}$.

Em nosso estudo, dos 16 pacientes com diagnóstico de flail ao ETE, submetidos a cirurgia, o diagnóstico foi confirmado em todos os casos.

Embora a detecção da regurgitação mitral seja acurada também aoETT, podem ocorrer discrepâncias significativas

\begin{tabular}{|c|c|c|}
\hline \multicolumn{3}{|c|}{ Tabela I - Dados clínicos de 35 portadores de flail mitral valve } \\
\hline Variáveis & $\begin{array}{l}\text { Tratamento clínico } \\
\qquad(\mathrm{n}=19)\end{array}$ & $\begin{array}{c}\text { Tratamento cirúrgico } \\
(\mathrm{n}=16)\end{array}$ \\
\hline Idade média (anos) & 71 & 57 \\
\hline Sexo (masculino/feminino) & $16 / 3$ & $11 / 5$ \\
\hline \multicolumn{3}{|l|}{ Etiologia } \\
\hline Prolapso & 8 & 8 \\
\hline Degenerativa & 6 & 4 \\
\hline Isquêmica & 3 & 2 \\
\hline Reumática & 2 & 2 \\
\hline Endocardite & 2 & 1 \\
\hline \multicolumn{3}{|l|}{ Grau da regurgitação mitral } \\
\hline Moderado & 7 & 3 \\
\hline Importante & 12 & 13 \\
\hline $\begin{array}{l}\text { Tempo médio de } \\
\text { acompanhamento (dias) }\end{array}$ & 250 & 514 \\
\hline \multicolumn{3}{|c|}{ Classe funcional pós-tratamento (NYHA) } \\
\hline I & 7 & 11 \\
\hline II & 3 & 2 \\
\hline \multicolumn{3}{|l|}{ Óbito } \\
\hline Intra-hospitalar & 6 & 3 \\
\hline Pós-hospitalar & 3 & 0 \\
\hline
\end{tabular}

com relação a esses dois métodos, principalmente quando a regurgitação mitral não é importante ${ }^{7}$. Conseqüentemente, a sensibilidade do ETE é significativamente mais elevada (100\%) do que a do ETT, tanto na avaliação acurada do grau da regurgitação mitral quanto do mecanismo do flail $^{8}$. Porém, como não foi feita comparação entre o grau de regurgitação mitral ao ETT e ao ETE, a maior sensibilidade do ETE não pôde ser demonstrada neste estudo.

A regurgitação mitral é uma alteração valvar comume, freqüentemente, difícil de se tratar, pois o curso clínico desta doença pode permanecer inalterado por vários anos. A correção cirúrgica pode aliviar os sintomas, porém, quandoé realizada em pacientes sintomáticos já com função sistólica ventricular comprometida, estes evoluem com ICC no pósoperatório e apresentam prognóstico ruim ${ }^{3}$. Assim, a correção cirúrgica deve ser realizada precocemente, seguramente antes de se instalar a disfunção do VE. Estudos prévios da história natural da regurgitação mitral ${ }^{3}$ oferecem pouca informação sobre a morbidade e relatam taxas variadas de mortalidade. Sobrevida em cinco anos de 27 a $97 \%$ tem sido relatada. Esta ampla variação observada nos estudos pode ser o resultado de graus pouco definidos de regurgitação, estudos contendo número pequeno de pacientes e presença de condições coexistentes agravantes, como endocardite, insuficiência coronária, e outras, associada à regurgitação mitral.

Neste estudo, dos 35 pacientes com diagnóstico de flail, 19 foram submetidos a tratamento clínico e os 10 sobreviventes estão em CF I ou II. Este achado é semelhante ao observado em um estudo do acompanhamento de 29 pacientes durante 19 meses, em que, de 15 pacientes não operados, 13 estavam em CF I ou II e, apenas dois, em CF III ${ }^{7}$. Os pacientes podem evoluir bem inicialmente, apesar da gravidade da regurgitação mitral. Contudo, a morbimortalidade a longo prazo é elevada. Em importante estudo realizado na Clínica Mayo, em 229 pacientes com diagnóstico de flail mitral valve, a taxa anual de mortalidade dos pacientes tratados clinicamente foi superior a dos pacientes submetidos a cirurgia ${ }^{3}$. Neste estudo, após 10 anos de acompanhamento, $63 \%$ dos pacientes encontravam-se em ICC, $30 \%$ apresentavam fibrilação atrial e $90 \%$ tinham sido submetidos a cirurgia valvar ou evoluído para óbito, demonstrando assim a alta morbimortalidade desta doença a longo prazo.

A ruptura, portanto, pode se apresentar tanto com curso clínico agudo, subagudo ou crônico. Como a função ventricular esquerda deteriora insidiosamente, o paciente pode evoluir bem nos primeiros anos. Contudo, o prognóstico a longo prazo seguramente parece ser melhor nos pacientes submetidos a cirurgia.

A baixa $\mathrm{CF}$ encontrada nos sobreviventes do presente estudo pode ser explicada pela alta mortalidade inicial devido às condições associadas (selecionando desta forma pacientes menos graves para o seguimento) e, também, pela característica insidiosa da evolução clínica da doença. O período de acompanhamento deste estudo é curto para se avaliar as consequiências da regurgitação mitral a longo prazo.

Estudos recentes sugerem que o ecocardiograma tridi- 
mensional possa acrescentar informações ao ETE e ajudar o cirurgião a determinar a estratégia operatória, especialmente na reconstrução valvar ${ }^{13}$. Contudo, a ruptura de cordoalha pode não ser visualizada por essa técnica e, portanto, a sensibilidade do método nesta detecção pode ser menor do que a do ETE bidimensional ${ }^{7}$.

Em conclusão, o ETE representa um excelente método para o diagnóstico da má coaptação dos folhetos da valva mitral e da ruptura de cordoalha tendínea, assim como da condição patológica subjacente. A regurgitação mitral também é avaliada adequadamente por esta metodologia.

A mortalidade à época do diagnóstico pode ser elevada, geralmente devido às doenças associadas. Apesar da gravidade da regurgitação mitral, alguns pacientes podem permanecer assintomáticos ou oligossintomáticos por anos antes do aparecimento da deterioração da função ventricular. Portanto, a baixa CF observada nestes pacientes pode se dever ao período relativamente curto de seguimento.

\section{Referências}

1. Pearson AC, Vrain JC, Mrosek D, Labovitz AJ - Color Doppler echocardiographic evaluation of patients with a flail mitral leaftlet. J Am Coll Cardiol 1990; 1: 232-9.

2. Himelman RB, Kusumoto F, Oken K et al - The flail mitral valve: echocardiographic findings by precordial and transesophageal imaging and Doppler color flow mapping. J Am Coll Cardiol 1991; 1: 272-9.

3. Ling LH, Eniquez-Sarano M, Seward JB et al - Clinical outcome of mitral regurgitation due to flail leaflet. N Engl J Med 1996; 335: 1417-23.

4. Sweatman T, Selzer A, Kamagaki M, Cohn K-Echocardiographic diagnosis of mitral regurgitation due to ruptured chordae tendineae. Circulation 1972; 46: 580-6.

5. Hozami T, Yoshikawa J, Yoshida K, Yamaura Y, Akasaka T, Shakudo M-Direct visualization of ruptured chordae tendineae by transesophageal two-dimensional echocardiography. J Am Coll Cardiol 1990; 16: 1315-9.

6. Sanders CA, Austen WG, Harthorne JW, Dinsmore RE, Scannell JG-Clinical outcome of mitral regurgitation due to flail leaflet. NEngl J Med 1967; 276: 943-9.

7. DePace NL, Mintz GR, Ren JF et al - Diagnosis and surgical treatment of mitral regurgitation secondary to ruptured chordae tendineae. Am J Cardiol 1983; 52: 789-95.
8. Schluter M, Langenstein BA, Hanrath P, Kremer P, Bleifeld W-Assessment of transesophageal pulsed Doppler echocardiography in the detection of mitral regurgitation. Circulation 1982; 66: 784-9.

9. Habbad MA, Haff JL - Characteristic pulsed Doppler findings in patients with flail mitral valve, Chest 1987; 91: 571-4

10. Seward JB, Khandheria BK, Edwards WD, Freeman WK, Tajik AJ - Biplanar transesophageal echocardiography: anatomic correlations, image orientation, and clinical applications. Mayo Clinic Proc 1990; 65: 1193-1213.

11. Child JS, Skorton DJ, Tylor RD et al - M mode and cross-sectional echocardiographic features of flail posterior mitral leaflets. Am J Cardiol 1979; 44: 1383-9.

12. Waller BF, Morrow AG, Maron BJ et al - Etiology of clinically isolated, severe, chronic, pure mitral regurgitation: analysis of 197 patients over 30 years of age having mitral valve replacement. Am Heart J 1982; 104: 276-88.

13. Binder T, Globits $\mathrm{S}$, Zangeneh $\mathrm{M}$ et al - Value of three-dimensional echocardiography as un adjunct to conventional transesophageal echocardiography. Cardiology 1996; 87: 335-42. 